\title{
Increased Incidence and Prevalence of Cardiovascular Events and Mortality in Female Patients with NAFLD: A Meta-Analysis and Meta-Regression
}

\author{
Yaser Khalid, DO ${ }^{1^{*}}$ (D) Neethi Dasu, DO ${ }^{1}$ (D) Herman Suga, DO ${ }^{1}$, Kirti Dasu, BA ${ }^{2}$, Debashis Reja, \\ $M D^{3}$, Ankit Shah, DO ${ }^{4}$, Donald McMahon, $\mathrm{DO}^{5}$ and Adam Levine, DO 6
}

\author{
${ }^{1}$ Division of Internal Medicine, Rowan University School of Medicine at Jefferson Health System, USA \\ ${ }^{2}$ Division of Biology, Syracuse University, USA \\ ${ }^{3}$ Division of Internal Medicine, Rutgers Robert Wood Johnson Medical School, USA \\ ${ }^{4}$ Division of Cardiovascular Medicine, Rowan University School of Medicine at Jefferson Health System, USA \\ ${ }^{5}$ Division of Gastroenterology, Rowan University School of Medicine at Jefferson Health System, USA \\ ${ }^{6}$ Division of Interventional Cardiology, Virtua Lourdes Health System, USA
}

*Corresponding author: Yaser Khalid, DO, Division of Internal Medicine, Rowan University School of Medicine at Jefferson Health System, Stratford, New Jersey, USA

\begin{abstract}
Background: Non-alcoholic fatty liver disease (NAFLD) and cardiovascular diseases (CVD) share similar risk factors. Recent studies have focused on obesity and insulin-resistance, but the link between NAFLD and CVD persists regardless of traditional risk factors. Despite the increased incidence and prevalence of NAFLD world-wide, there has been no thorough investigation of gender disparities nor a closer look taken into investigating the role gender may play in increased cardiovascular (CV) mortality incidence and prevalence in patients with NAFLD.
\end{abstract}

Objective: We assessed incidence and prevalence of $\mathrm{CV}$ events and mortality based on gender in patients with NAFLD, at any stage of fibrosis. A meta-regression was conducted to further analyze the impact of age on both genders.

Methods: An aggregate analysis was performed on ten studies with NAFLD patients. A random-effects model was used to pool the overall incidence and prevalence rates of CV events and mortality as well as all-cause mortality to examine any gender disparity. We also performed a meta-regression analysis to evaluate the effect of age on mortality for men versus women with NAFLD and CV events and mortality. Summary odds ratios (OR) and 95\% confidence intervals $(\mathrm{Cl})$ were estimated using a random-effects model.

Results: In 259,598 patients with NAFLD, of which $44 \%$ were females and $56 \%$ were males, all-cause mortality was
$1.5 \times$ higher in women compared to men (OR 1.65, 95\% Cl $1.12-2.43, p<0.012)$. CV events and mortality were also 2 $x$ higher in women compared to men (OR $2.1295 \% \mathrm{Cl} 1.65-$ $2.73, p<0.001)$. On meta-regression, females had higher mortality with advancing age starting at age 42 (coefficient $=$ 0.0518, $p=0.00001$ ).

Conclusion: For patients with NAFLD, women had a markedly higher incidence and prevalence of CV events, CV mortality and all-cause mortality when compared to men. Meta-regression showed increased mortality among women with advancing age. As the incidence and prevalence of NAFLD and concomitant CV events increases worldwide, we urge the medical community to increase surveillance and perform rigorous cardiovascular risk assessments for women, especially beginning at age 42. Additionally, we recommend heterogenous surveys of gender disparities, increased focus on gender as a decisive factor for downstream CV events, the relationship between NAFLD severity and gender-based mortality differences, and larger studies representing equivalent male and female populations.

\section{Keywords}

NAFLD, CVD, CAD, Gender, Women's health, Cardiovascular mortality

Citation: Khalid Y, Dasu N, Suga H, Dasu K, Reja D, et al. (2020) Increased Incidence and Prevalence of Cardiovascular Events and Mortality in Female Patients with NAFLD: A Meta-Analysis and Meta-Regression. Int J Clin Cardiol 7:187. doi.org/10.23937/2378-2951/1410187

Accepted: July 08, 2020; Published: July 10, 2020

Copyright: (c) 2020 Khalid Y, et al. This is an open-access article distributed under the terms of the Creative Commons Attribution License, which permits unrestricted use, distribution, and reproduction in any medium, provided the original author and source are credited. 


\section{Introduction}

Approximately 90 million adults in the United States are diagnosed with nonalcoholic fatty liver disease (NAFLD), which is emerging as one of the leading causes of chronic liver disease [1-3]. NAFLD encompasses a spectrum of diseases that histologically varies from mild steatosis or steatohepatitis that can advance to fibrosis, cirrhosis, and eventually hepatocellular carcinoma [15], NAFLD results from causes not attributed to alcohol consumption, viruses, drugs, toxins, and autoimmune diseases [1,2].

Cirrhosis was initially believed to be protective against coronary artery disease (CAD), but recent studies have demonstrated a strong correlation between cardiovascular disease (CVD) and the development of NAFLD, and is also the leading cause of death in patients with NAFLD [1,5-10]. NAFLD and cardiovascular disease share common risk factors such as: Hypertension, hyperlipidemia, diabetes, tobacco use, unhealthy diets, sedentary lifestyles, obesity, presence of post-transcriptional regulators in microRNAs, increased expression of the GCKR gene, and increased abdominal fat distribution [6,8-14]. As a result, a common pathophysiological pathway also exists via insulin resistance and inflammation and oxidative stress pathways $[12,15]$. NAFLD and CVD have increased Framingham risk scores and reduced life expectancy which contributes to higher mortality than the general population [16]. CV complications in patients with NAFLD are the following: CAD, subclinical atherosclerosis, cardiac arrhythmias, and structural and functional abnormalities [6].

Since 1997 to the present, CVD has been the leading cause of death for women worldwide, accounting for $43 \%$ fatalities of US females. Despite such a strong association between NAFLD and CVD, gender differences have rarely been studied [17]. Women are underrepresented in most clinical trials and there is little information outlining the differences in care between the genders [18]. In this paper we discuss the association of NAFLD with cardiovascular disease in men and women, the mechanisms underlying this association, and the differences between the genders with regards to age and incidence.

\section{Methods}

\section{Search methods and study selection}

A systematic review and meta-analysis of the literature was conducted by the principles set in the Preferred Reporting Items for Systematic Reviews and Meta-Analyses: the PRISMA Statement. Two co-authors (YSK and NRD) independently searched published studies indexed in OVID, Cochrane Central Register of Controlled Trials (CENTRAL) via the Wiley Interface, Web of Science Core Collection, MEDLINE, EMBASE, and Google Scholar from beginning of the project on October 1,
2019 to January 30, 2020. We searched the terms "cardiovascular diseases" and "cardiovascular mortality" in all fields with each of the following words: "non-alcoholic fatty liver disease", "non-alcoholic steatohepatitis", " "non-alcoholic fatty liver disease", "nonalcoholic fatty liver disease," "fatty liver", "liver fat", "steatosis", "NAFLD", "NASH", "women," "gender," "all-cause mortality." Terms used to define the outcomes included "prevalence" and "incidence." All electronically published papers were screened by titles or abstracts. We only reviewed entire papers if they were pertinent for inclusion for our study. All detected references were saved electronically in the Zotero reference management program and all duplicates were identified and removed. The systematic search was supplemented by manual review of all references in the retrieved eligible studies.

Randomized, prospective, retrospective, cross-sectional, or nonrandomized controlled studies were considered for inclusion if they reported the incidence of cardiovascular disease and mortality in patients with NAFLD. The inclusion criteria were as follows: 1) Observational studies (case-control, cross-sectional, or cohort studies) published as original studies to evaluate the association between cardiovascular disease and mortality and NAFLD; 2) Odds ratios (ORs), relative risk (RRs), hazard ratio (HRs) or standardized incidence ratio (SIRs) with $95 \%$ confidence intervals (Cls) were provided; 3 ) Non-NAFLD participants were used as the reference group in cross-sectional and cohort studies, and participants without cardiovascular disease and/or mortality were used as the reference group in case-control study. When the needed data was not directly found in the published articles, we obtained the necessary data from the authors through electronic communication or via reviewing their supplemental reports.

We excluded all studies that did not meet these criteria or that reported insufficient data. We used the checklist for Critical Appraisal and Data Extraction for Systematic Reviews of Prediction Modeling Studies (CHARMS) for study quality assessment. The search methods, study selection/eligibility criteria are outlined in Figure 1 [19].

\section{Data extraction}

A standardized data collection form was used to extract the following information: Last name of the first author, title of the article, study design, year of study, country of origin, year of publication, sample size, characteristics of included participants, evaluation of NAFLD through liver ultrasound, liver function tests (AST, ALT, GGT) or liver biopsy; evaluation of subclinical atherosclerosis (pathological carotid intima media thickness or presence of carotid plaques); and evaluation of CAD (presence of at least $50 \%$ stenosis at one or more major coronary arteries) were recorded. The table was com- 


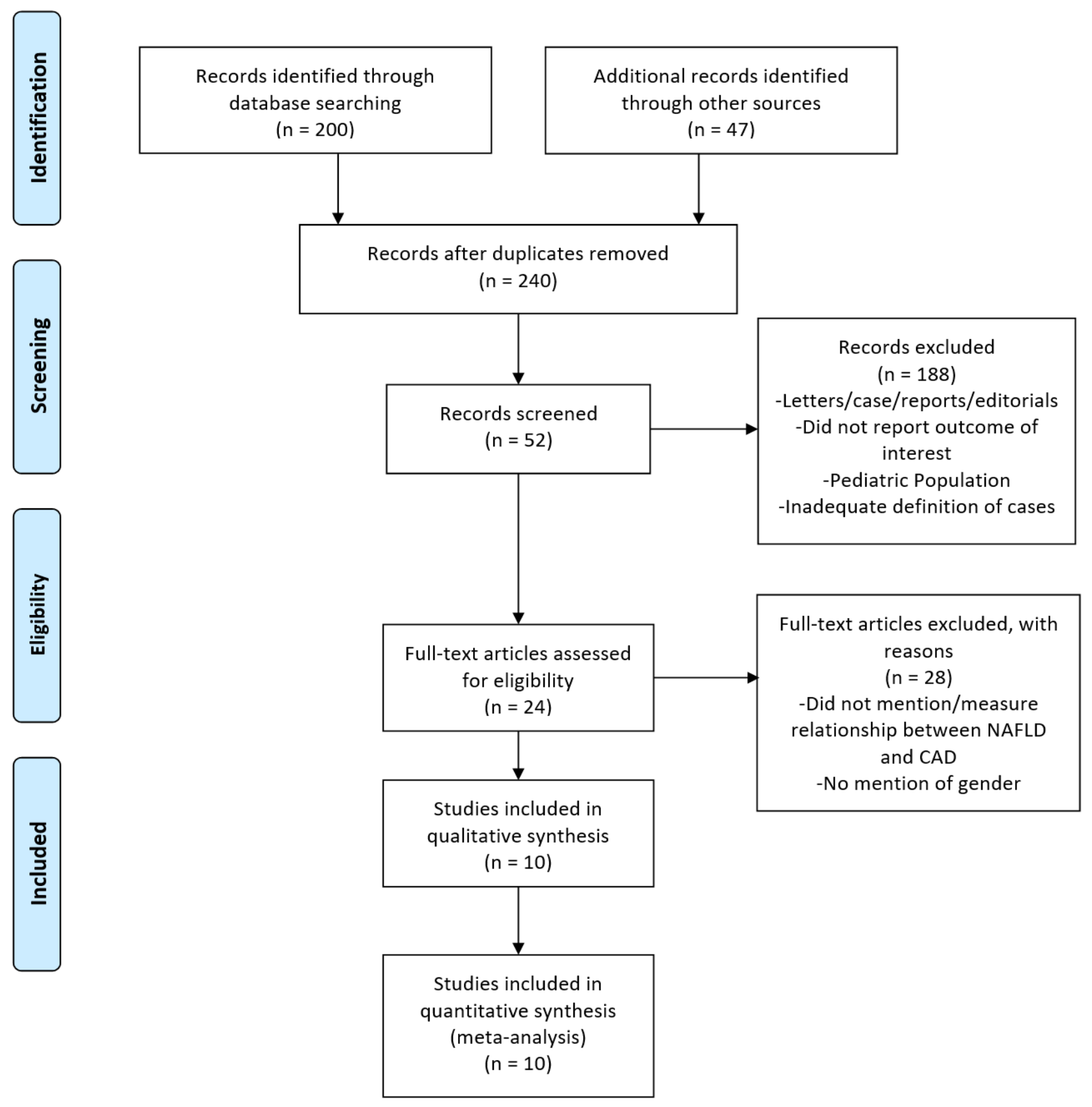

Figure 1: Prisma flow chart of studies screened and included in meta-analysis.

pleted by the first author and verified by an additional member of the study team. The two investigators mentioned performed independent data extraction.

\section{Meta-analysis}

The Hartung-Knapp-Sidik-Jonkman (HKSJ) method was employed to complete the statistical data using MedCalc software with: 1) A summary of data from individual studies; 2) An investigation of the studies heterogeneity graphically and statistically; 3 ) Calculation of clustered indexes; 4) Exploration of heterogeneity; and 5) Graphical illustration via Forest Plots. Our assumption of heterogeneity was tested for each planned analysis using the Cochrane- $Q$ heterogeneity and $\mathrm{I}^{2}$ statistics with each of the following values: Low $0-25 \%$, moderate 25 $75 \%$, and high $>75 \%$. Random effects models using the Mantel-Haenszel method and fixed effects models were used. Meta-regression was analyzed using a generalization of Littenberg and Moses Linear model weighted by inverse of the variance or study size or unweighted. A secondary analysis was performed using the Der Simonian \& Laird method and fixed effects models through Comprehensive Meta-Analysis software. Heterogeneity was assessed using the Chi-square test and $\mathrm{I}^{2}$ statistic.

\section{Study quality and publication bias}

Newcastle-Ottawa scale (0 to 9 points) (Appendix A), using the methodology described by Downs and Black, was utilized to quantify study quality. All statistical tests were two-tailed and the type I error rate was set at $5 \%$. Only two-sided tests with a significance level of 0.05 were used. Confidence intervals ( $\mathrm{Cls}$ ) of individual studies were calculated. A sensitivity analysis was also conducted. Publication bias was assessed using funnel plots 


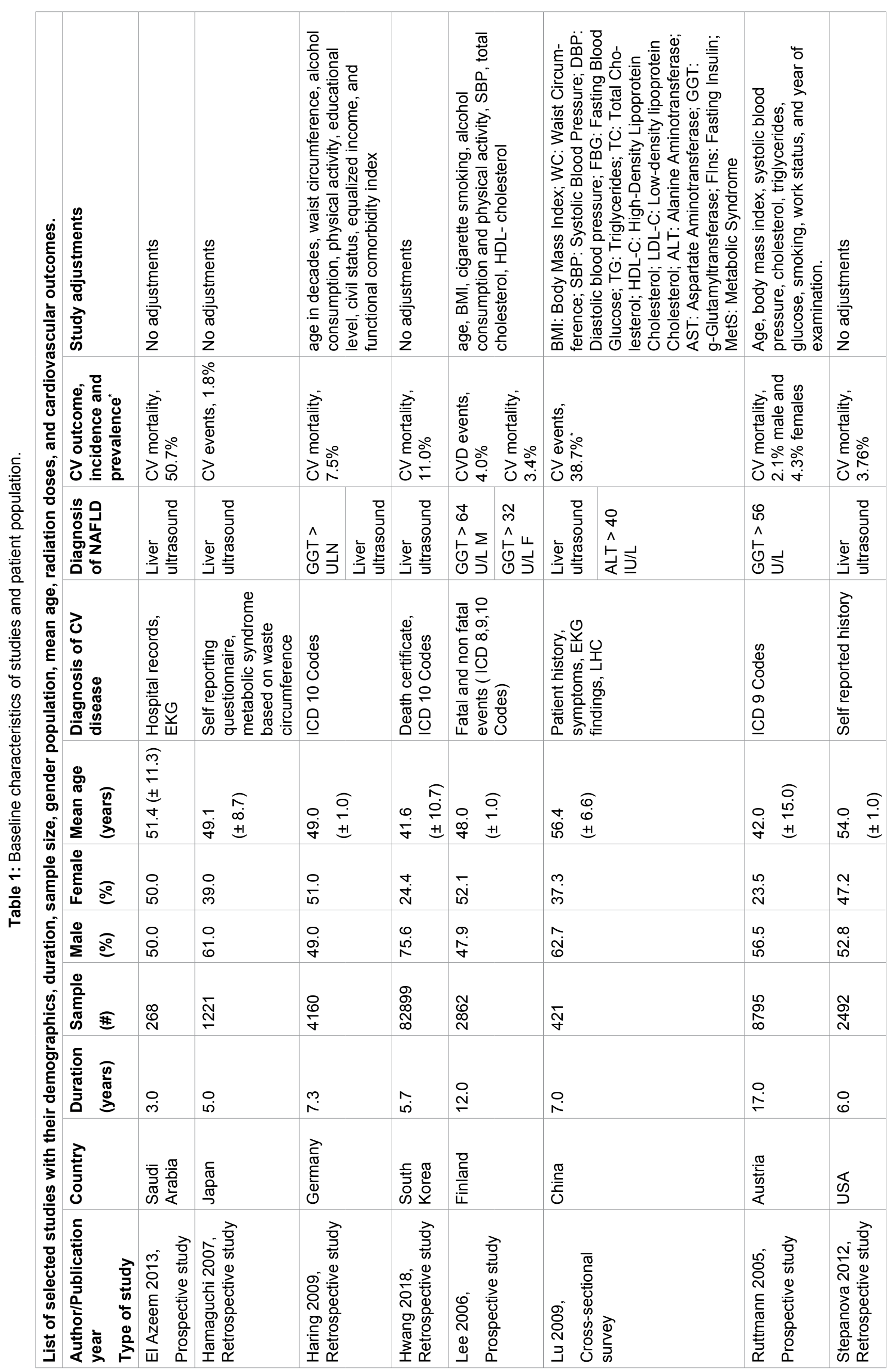




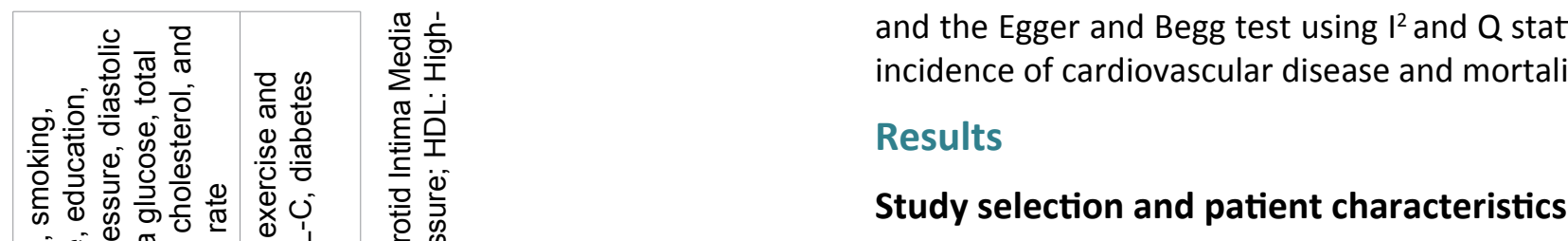

The process of study selection is presented in Figure 1. Our initial literature search yielded 247 potential studies for review. Following exclusion of review articles, case reports, retrospective studies, abstracts, studies with insufficient data, and articles with overlapping study populations and redundant data, a total of 10 studies were included in the final analysis (Table 1 ). All studies were conducted in the USA, Europe, Japan, Saudi Arabia, South Korea, and China. 8 studies reported incidence, 4 of which were retrospective and 4 were prospective. 2 studies were cross-sectional studies that reported prevalence. The number of patients in each study varied with over 80,000 patients in the largest study and 268 patients in the smallest study. Among 259,598 patients with NAFLD, $44 \%$ were females and $56 \%$ were males, with a weighted mean age of 50 years.

\section{Defining outcomes}

Asymptomatic atherosclerosis and coronary artery disease were evaluated in a selection of studies that reported carotid intima media thickness, duplex arterial ultrasound for peripheral vascular disease, aortic plaque on abdominal ultrasound, coronary computed tomography angiography (CCTA) findings of plaque (at least $50 \%$ stenosis of at least one of the major coronary arteries), exercise or nuclear stress testing results, coronary angiography results (at least $50 \%$ stenosis of at least one of the major coronary arteries), and carotid plaques (measured by ultrasound). Cardiovascular events were defined as asymptomatic atherosclerosis and coronary artery disease. CVD was defined as symptomatic cardiovascular events resulting in hospitalization and morbidity as the end-point. Cardiovascular mortality was defined as death from one of the following causes: Stroke or transient ischemic attack, $\mathrm{Ml}$, acute coronary syndrome (including unstable angina), and heart failure which had required hospitalization.

NAFLD also has a variety of definitions in the literature and the only gold standard for diagnosis is biopsy, which is not always available for diagnosis. In our studies, NAFLD was diagnosed by liver ultrasound, liver function tests, and liver biopsy (when possible).

\section{Incidence and prevalence of cardiovascular events and mortality}

The cumulative incidence and prevalence of cardiovascular events and mortality was found to be 2-times higher in women compared to men with known NAFLD (OR $2.1295 \% \mathrm{Cl}$ 1.65-2.73, $\mathrm{p}<0.001$ ) (Figure 2). Allcause mortality was also 1.5-times higher incidence and prevalence of all-cause mortality in women compared 


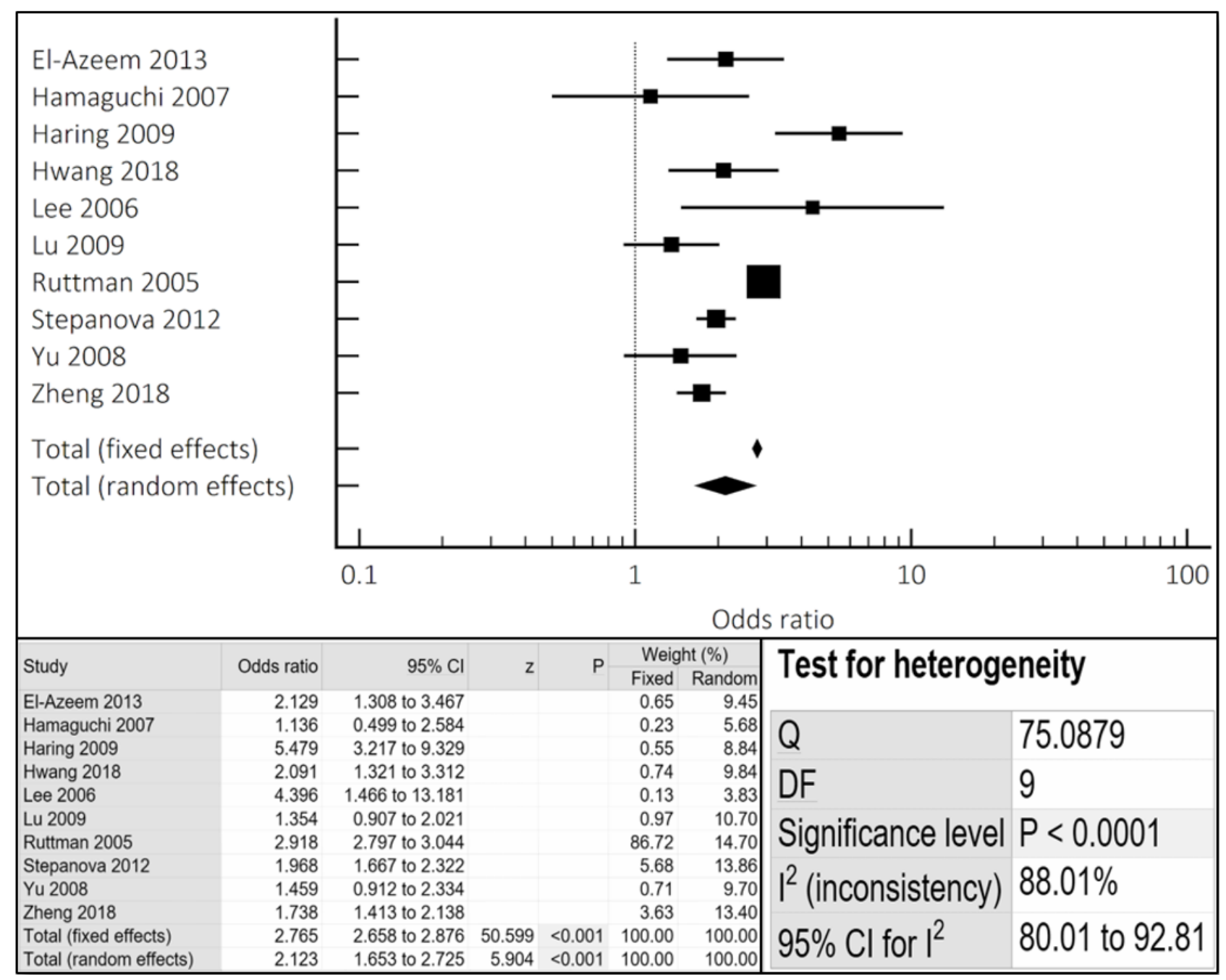

Figure 2: Cumulative incidence and prevalence of CV events/mortality noted in NAFLD females compared to males via combined fixed and random effects.

Odds ratio, confidence interval, and weight of studies for NAFLD females compared to males for cardiovascular mortality via combined fixed and random effects models. Test for heterogeneity of studies.

to men with NAFLD (OR 1.65, 95\% Cl 1.12-2.43, p < 0.012) (Figure 3). Similar results were noted both in the fixed and random effect models.

On univariate meta-regression (Figure 4), when plotting log odds ratio of cardiovascular events and mortality among males versus females ( $y$-axis) against age ( $x$-axis), women had higher mortality with advancing age (regression coefficient $=0.0518, p=0.00001$ ). $Q$ and $I^{2}$ statistics were analysed to assess heterogeneity; the results of which can be found in Appendix B.

Funnel plot analysis (Appendix C, Appendix D and Appendix E) did not reveal asymmetry around the axis for the treatment effect in the assessed outcomes $(p<$ 0.05 by Begg and Mazumdar's test or Egger's test), suggesting lack of publication bias.

\section{Discussion}

In this meta-analysis, meta-regression, and systematic review, the impact of gender on both the incidence and prevalence of cardiovascular disease and mortality for adult patients with NAFLD was evaluated. The main findings of our study are the following: i) Female patients were found to have greater incidence and prevalence of cardiovascular events and mortality; ii) All-cause mortality incidence and prevalence was higher for female patients; iii) Men and women had a higher incidence and prevalence of mortality with advancing age, however, it is important to note that mortality incidence and prevalence was higher for women. This is the first systematic review, meta-regression, and meta-analysis assessing the role of gender in cardiovascular disease and mortality for patients with NAFLD. These findings illustrate the need for future investigations and the development of new screening guidelines for women as they are an understudied patient population.

NAFLD is a multifactorial disease that develops from the interactions of genetics, diet, and lifestyle $[5,6,20,21]$. NAFLD and CVD both have significant similarities in terms of risk factors, genetic predispositions, and pathophysiology. NAFLD has been associated with poor coronary artery collateral blood flow, insulin resistance, and systemic inflammation, which are all major contributors to CVD $[8,22]$. This meta-analysis revealed 


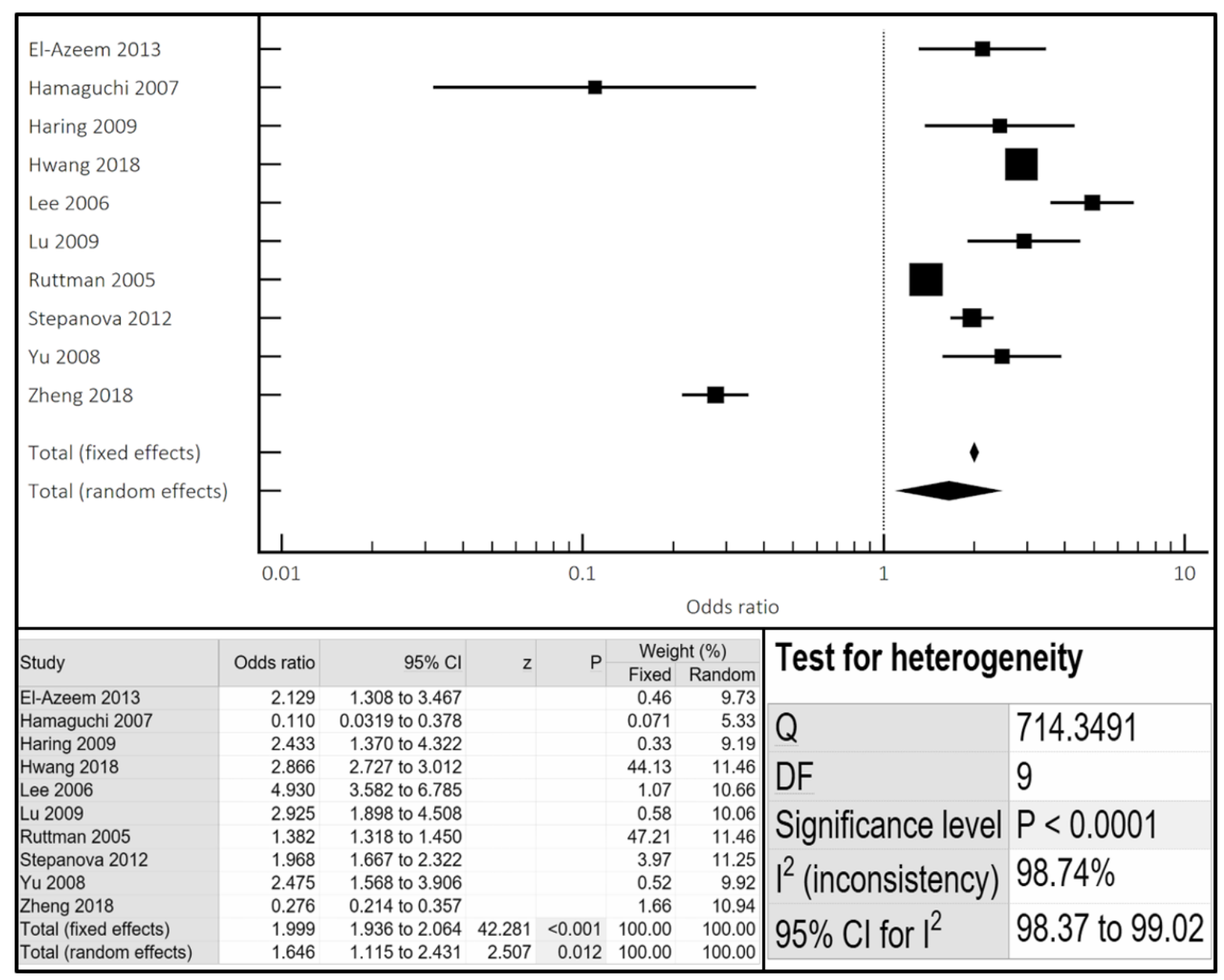

Figure 3: Cumulative incidence and prevalence of all-cause mortality noted in NAFLD females compared to males via combined fixed and random effects.

Odds ratio, confidence interval, and weight of studies for NAFLD females compared to males for all-cause mortality via combined fixed and random effects models. Test for heterogeneity of studies.

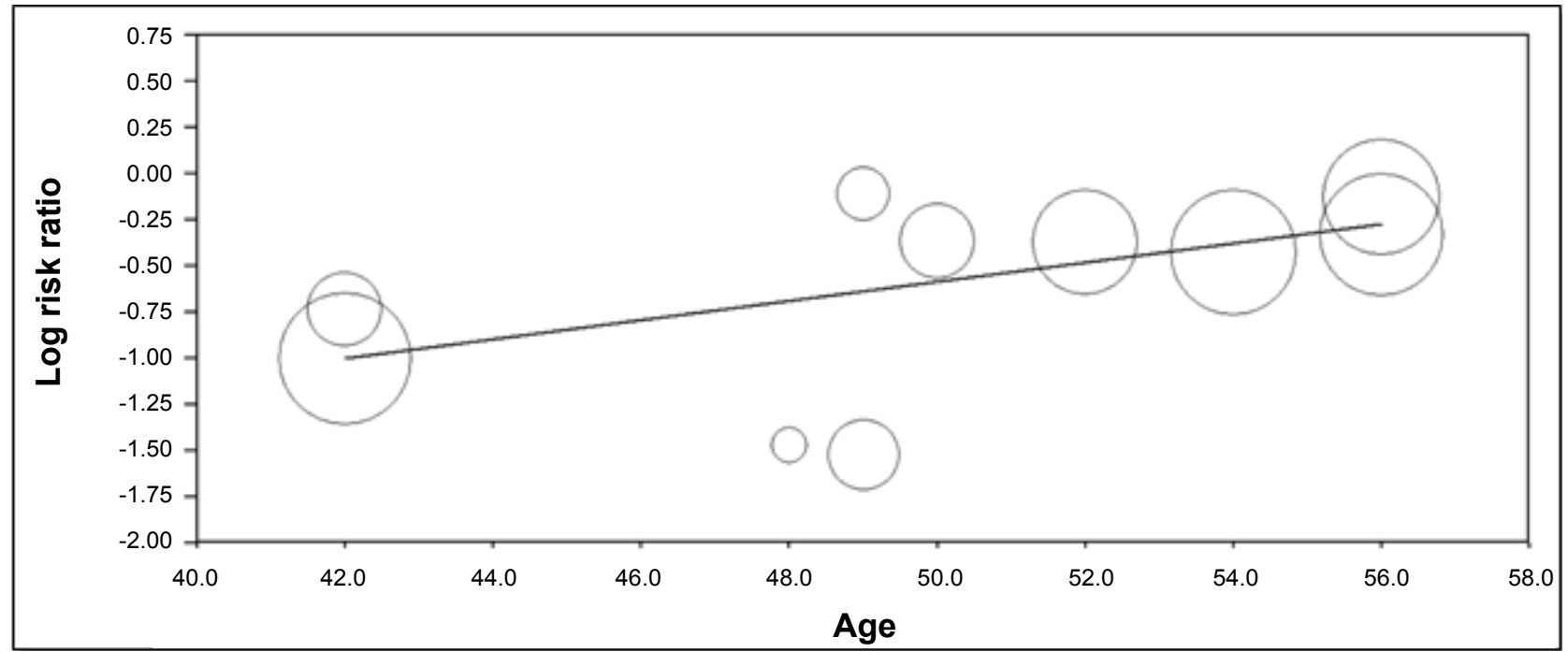

Figure 4: Meta-regression to assess for incidence and prevalence of CV events/mortality of NAFLD female patients with aging.

a 2-fold increased incidence of CV events and mortality in females when compared to male NAFLD patients, which further increased with aging (beginning at age 42)
(Figure 2 and Figure 4).

Our study identifies that the female gender has a strong yet independent impact on cardiovascular 
events and mortality. Early and aggressive screening must be considered for female patients given that patients with NAFLD have a higher prevalence of carotid atherosclerosis before cardiovascular events, when compared to the general population [23]. Previous studies on patients with both NAFLD and CVD do not analyze the effects of gender on the incidence of cardiovascular events and mortality. Late presentation of these diseases leads to longer ischemic exposure and thus increases the incidence of complications that may affect patients with both NAFLD and CVD [24,25]. Women are underrepresented in most pharmacotherapy trials but data and trials claim equal efficacy for both sexes. In our study, females only represented $44 \%$ of the patient population [26] and as a result, we focused on gender to elucidate the disparities between the two populations. We strongly suggest researchers to focus on gender disparity analysis in future trials to further elucidate the observed and clinically-relevant differences that arise from the statistical analysis.

Based on current literature, there appears to be a very definitive relationship between NAFLD and a higher risk of CVD that can be seen with imaging $[2,3,7]$. In this analysis of 10 studies, the initial definition of CVD varied by author. Zheng, et al. defined CV disease as carotid intima media thickening $>0.8 \mathrm{~mm}$ along with post-exercise brachial-ankle pulse wave velocity as surrogates for clinical cardiovascular disease [20]. Yun, et al., Harring, et al. and Hwang, et al. defined cardiovascular mortality via ICD 10 codes. Ruttman, et al. similarly defined CV disease via ICD 9 codes. Stepanova, et al. utilized patient's self reporting of their history of CV disease. Lu, et al., however, used history, symptoms, EKG, and coronary angiography findings. Lee, et al. utilized ICD 8, 9, and 10 codes of fatal and non-fatal CV events. Hamaguchi, et al. used survey answers and defined metabolic syndrome using waist circumference. El-Azeem, et al. used hospital records and EKG findings. Although carotid intima media thickness, symptoms, EKG, and coronary angiography findings are markers that may not necessarily correlate to clinical relevance and significance, they could be an early indicator of cardiovascular disease and should be monitored closely by clinicians. In several studies, NAFLD has been shown to be an independent predictor of a high plaque burden on CCTA in asymptomatic patients [27]. Despite being asymptomatic, these patients may develop clinically significant CVD thus highlighting the importance of monitoring our markers of CVD in NAFLD patients and potentially preventing future CV events.

With regards to the diagnosis of NAFLD, the majority of studies analyzed did not use liver biopsy to diagnose NAFLD. Liver ultrasound findings were considered adequate evidence of NAFLD. Some studies only used elevated liver biomarkers such as ALT and GGT greater than two-thirds the upper limit of normal to diagnose NAFLD, when all other etiologies of liver disease, such as alcohol, genetic, viral, and autoimmune causes were ruled out (Table 1 ). Ideally, the studies should have diagnosed NAFLD using liver biopsies - the gold standard - to achieve uniformity. However, biopsies are invasive procedures with their own sets of complications and are avoided, as a result, for diagnosing NAFLD or considered unnecessary when NAFLD is identified through ultrasounds or biomarkers. Thus, we recommend earlier preliminary screening of patients who have metabolic syndrome or other risk factors for CV disease via the use of liver indexes Fibrosis-4 score, FibroScan or NAFLD fibrosis score [28].

Our study further revealed an increased incidence and prevalence of all-cause mortality in the female population of NAFLD (Figure 3). In a meta-analysis of 40 studies by Muso, et al. it was reported that NAFLD increased the risk of mortality due to the liver by up to 10-fold [29]. This extensive increase in mortality may have been attributable to liver fibrosis as the underlying etiology. Ekstedt, et al. established that hepatic fibrosis stage was the strongest predictor for all-cause in patients with histologically confirmed NAFLD over 26.4 years [29]. Having additional data in terms of the degree of liver fibrosis and disease stage could have helped us further understand why NAFLD increases all-cause mortality for this patient population. We would then be able to analyze the impact of the degree of liver fibrosis and disease severity on outcomes. In 2017, patients with NAFLD accounted for an alarming $10 \%$ of patients undergoing liver transplantation in the United States [30]. Our study reinforces the need for further investigation of NAFLD related all-cause mortality in terms of gender differences.

Our meta-regression analysis revealed that advancing age (beginning at age 42) was associated with higher cardiovascular mortality in both men and women, but a higher incidence and prevalence of mortality was noted for women with advancing age when compared to men. This gender disparity is very critical to note as women tend to present later in the course of NAFLD and CVD possibly due to the presence of atypical symptoms which can delay diagnosis and treatment [31,32]. Increasing age has been persistently identified as a risk factor for fibrotic progression to cirrhosis throughout multiple studies and there have been several theories about why females with NAFLD have increased mortality with advancing age [33]. One theory postulates that older postmenopausal females have ovarian senescence secondary to estrogen deficiency which may be associated with severe steatosis and fibrosing NASH [33]. Ludwig, et al. reported that NAFLD was common among women older than 40 with metabolic comorbidity and thus leading to a higher mortality [34]. Interestingly, per earlier studies, men have an increasing prevalence of NAFLD from early to middle-age which starts declining after age 50, whereas the opposite is true for women. Women tend to have lower prevalence of NAFLD until the fifth de- 
cade of life, and peak during the sixth decade $[35,36]$. Our meta-regression substantiates these findings from earlier studies and this may contribute to the increased all-cause and cardiovascular mortality for females seen in our study.

\section{Study Limitations and Strengths}

Several strengths need to be recognized for our study. For our systematic review and meta-analysis, a variety of distinct reference databases were utilized. We also analyzed a large patient population.

Our meta-analysis has limitations. Although the quality assessment methods and bias assessments methods used in our studies were considered high quality and with minimal bias, the $Q$ and $I^{2}$ statistics determined a high degree of heterogeneity between the studies. Differences in the inclusion criteria, endpoint definitions, and human error associated with capture and confirmation of data are all potential confounding sources among studies that analyze registries. We could not access patient-level data, such as medications, laboratory data, and imaging, to allow adjustment for factors that might influence the incidence of cardiovascular events and mortality for patients with NAFLD. Finally, this review is limited by the classification and diversity of cardiovascular disease and coronary artery disease definitions. Our study results must be interpreted cautiously with respect to these myriad definitions. More research on the relationship between cardiovascular disease and NAFLD in the form of randomized clinical trials where both NAFLD and CVD pathology is defined and evaluated clearly is necessary to solidify our current findings. Additionally, because our findings reveal that both age and gender have precedence in terms of disease outcomes and mortality, the need for research specifically focused on the link between the two disease states and these parameters only increases as the incidence and prevalence rates of both diseases continues to rise.

\section{Conclusions}

It has been estimated that NAFLD will be the main etiology of end-stage liver disease of patients that are undergoing liver transplantation by the year 2025 [7]. The increased incidence and prevalence of mortality in female patients with both NAFLD and CVD cannot be overlooked and requires thorough investigations performed by large, multi-center trials. This can be further investigated in future studies in terms of pathophysiology and genetics of NAFLD and CVD [31,32]. For patients with NAFLD, women had a markedly higher incidence and prevalence of $\mathrm{CV}$ event-related mortality and allcause mortality when compared to men. Advancing age increased mortality among female patients with NAFLD. Our study suggests the importance of monitoring silent markers as well as early preventative medicine in female populations with NAFLD, due to their increased risk of developing CVD. We believe this can be achieved by encouraging this subset of patients to reduce certain risk factors that overlap in both disease processes such as weight and sedentary behavior to help improve overall metabolic health and decrease disease burden. Additionally changes to unhealthy dietary habits in favor of a more healthy diet (i.e. Mediterranean Diet) would help to further reduce the risk factors for this sub-population.

\section{Disclosures}

No conflict of interest.

No acknowledgements.

\section{References}

1. LB VanWagner (2018) New insights into the link between NAFLD and atherosclerotic coronary heart disease? J Hepatol 68: 890-892.

2. Tracey G Simon, Maria Esther Perez Trejo, Robyn McClelland, Ryan Bradley, Michael J Blaha, et al. (2018) Circulating interleukin- 6 is a biomarker for coronary atherosclerosis in nonalcoholic fatty liver disease: Results from the multi-ethnic study of atherosclerosis. Int J Cardiol 259: 198204.

3. LR Cuenza, TLJ Razon, JC Dayrit (2017) Correlation between severity of ultrasonographic nonalcoholic fatty liver disease and cardiometabolic risk among Filipino wellness patients. J Cardiovasc Thorac Res 9: 85-89.

4. Yoosoo Chang, Seungho Ryu, Ki-Chul Sung, Yong Kyun Cho, Eunju Sung, et al. (2019) Alcoholic and non-alcoholic fatty liver disease and associations with coronary artery calcification: Evidence from the Kangbuk Samsung Health Study. Gut 68: 1667-1675.

5. Seok-Hyung Kim, Hae Yeul Park, Hye Sun Lee, Kwon Soo Jung, Moon Hyoung Lee, et al. (2020) Association between non-alcoholic fatty liver disease and coronary calcification depending on sex and obesity. Sci Rep 10: 1025.

6. A Ismaiel, DL Dumitraşcu (2019) Cardiovascular risk in fatty liver disease: The liver-heart axis-literature review. Front Med 6: 202.

7. A Kadayifci, V Tan, PC Ursell, RB Merriman, NM Bass (2008) Clinical and pathologic risk factors for atherosclerosis in cirrhosis: A comparison between NASH-related cirrhosis and cirrhosis due to other aetiologies. J Hepatol 49: 595-599.

8. S Mendis, K Thygesen, K Kuulasmaa, S Giampaoli, M Mahonen, et al. (2011) World Health Organization definition of myocardial infarction: 2008-09 revision. Int J Epidemiol 40: 139-146.

9. EG Nabel, E Braunwald (2012) A tale of coronary artery disease and myocardial infarction. N Engl J Med 366: 54-63.

10. RA Harrington (2017) Targeting inflammation in coronary artery disease. N Engl J Med 377: 1197-1198.

11. AL Madika, C Mounier-Vehier (2016) La maladie coronaire de la femme: de vraies spécificités à bien connaître pour améliorer les prises en charge. La Presse Médicale 45: 577-587.

12. A Braza-Boïls, J Marí-Alexandre, P Molina, MA Arnau, M Barceló-Molina, et al. (2016) Deregulated hepatic microRNAs underlie the association between non-alcoholic fatty liver disease and coronary artery disease. Liver Int 36: 1221-1229. 
13. Y Gao, YC Wang, CQ Lu, C Zeng, D Chang, et al. (2018) Correlations between the abdominal fat-related parameters and severity of coronary artery disease assessed by computed tomography. Quant Imaging Med Surg 8: 579-587.

14. B Wang, F Li, J Guo, C Wang, D Xu, et al. (2019) Effects of liver function, insulin resistance and inflammatory factors on vascular endothelial dilation function and prognosis of coronary heart disease patients complicated with NAFLD. Exp Ther Med 17: 1306-1311.

15. T Vita, DJ Murphy, MT Osborne, NS Bajaj, A Keraliya, et al. (2019) Association between nonalcoholic fatty liver disease at CT and coronary microvascular dysfunction at myocardial perfusion PET/CT. Radiology 291: 330-337.

16. SE Kerut, JT Balart, EK Kerut, MR McMullan (2017) Diagnosis of fatty liver by computed tomography coronary artery calcium score. Echocardiography 34: 937-938.

17. EG Nabel (2000) Coronary heart disease in women - An ounce of prevention. N Engl J Med 343: 572-574.

18. J Prata, S Ramos, AQ Martins, F Rocha-Gonçalves, R Coelho (2014) Women with coronary artery disease: Do psychosocial factors contribute to a higher cardiovascular risk? Cardiol Rev 22: 25-29.

19. D Moher, A Liberati, J Tetzlaff, DG Altman, PRISMA Group (2009) Preferred reporting items for systematic reviews and meta-analyses: The PRISMA Statement. PLoS Med 6: e1000097.

20. T Niikura, K Imajo, A Ozaki, T Kobayashi, M Iwaki, et al (2020) Coronary artery disease is more severe in patients with non-alcoholic steatohepatitis than fatty liver. Diagnostics 10: 129.

21. $H$ Yang, D Li, X Song, F Liu, X Wang, et al. (2018) Joint associations of serum uric acid and ALT with NAFLD in elderly men and women: A Chinese cross-sectional study. J Transl Med 16: 285.

22. R Sao, WS Aronow (2018) Association of non-alcoholic fatty liver disease with cardiovascular disease and subclinical atherosclerosis. Arch Med Sci 14: 1233-1244.

23. S Petta, C Argano, D Colomba, C Cammà, VD Marco, et al. (2015) Epicardial fat, cardiac geometry and cardiac function in patients with non-alcoholic fatty liver disease: Association with the severity of liver disease. J Hepatol 62: 928-933.

24. B Safdar, ES Spatz, RP Dreyer, JF Beltrame, JH Lichtman, et al. (2018) Presentation, clinical profile, and prognosis of young patients with myocardial infarction with nonobstructive coronary arteries (MINOCA): Results from the VIRGO study. J Am Heart Assoc 7: e009174.
25. R Bugiardini, CNB Merz (2005) Angina with "normal" coronary arteries: A changing philosophy. JAMA 293: 477-484.

26. KA Liu, NA Dipietro Mager (2016) Women's involvement in clinical trials: Historical perspective and future implications. Pharm Prac 14: 708.

27. SB Lee, GM Park, JY Lee, BU Lee, JH Park, et al. (2018) Association between non-alcoholic fatty liver disease and subclinical coronary atherosclerosis: An observational cohort study. J Hepatol 68: 1018-1024.

28. ILK Nalbantoglu, EM Brunt (2014) Role of liver biopsy in nonalcoholic fatty liver disease. World J Gastroenterol 20: 9026-9037.

29. G Musso, R Gambino, M Cassader, G Pagano (2011) Meta-analysis: Natural history of non-alcoholic fatty liver disease (NAFLD) and diagnostic accuracy of non-invasive tests for liver disease severity. Ann Med 43: 617-649.

30. L Calzadilla-Bertot, GP Jeffrey, B Jacques, G McCaughan, M Crawford, et al. (2019) Increasing incidence of nonalcoholic steatohepatitis as an indication for liver transplantation in Australia and New Zealand. Liver Transpl 25: 25-34.

31. E Perdoncin, C Duvernoy (2017) Treatment of coronary artery disease in women. Methodist Debakey Cardiovasc $\mathrm{J}$ 13: 201-208.

32. AL Blomkalns, AY Chen, JS Hochman, ED Peterson, $K$ Trynosky, et al. (2005) Gender disparities in the diagnosis and treatment of non-ST-segment elevation acute coronary syndromes: Large-Scale Observations From the CRUSADE (Can Rapid Risk Stratification of Unstable Angina Patients Suppress Adverse Outcomes With Early Implementation of the American College of Cardiology/American Heart Association Guidelines) National Quality Improvement Initiative. J Am Coll Cardiol 45: 832-837.

33. S Ballestri, F Nascimbeni, E Baldelli, A Marrazzo, D Romagnoli, et al. (2017) NAFLD as a Sexual dimorphic disease: Role of gender and reproductive status in the development and progression of nonalcoholic fatty liver disease and inherent cardiovascular risk. Adv Ther 34: 1291-1326.

34. J Ludwig, DB McGILL, KD Lindor (1997) Review: Nonalcoholic steatohepatitis. J Gastroenterol Hepatol 12: 398-403.

35. JG Fan, J Zhu, XJ Li, L Chen, L Li, et al. (2005) Prevalence of and risk factors for fatty liver in a general population of Shanghai, China. J Hepatol 43: 508-514.

36. Y Eguchi, H Hyogo, M Ono, T Mizuta, N Ono, et al. (2012) Prevalence and associated metabolic factors of nonalcoholic fatty liver disease in the general population from 2009 to 2010 in Japan: A multicenter large retrospective study. J Gastroenterol 47: 586-595. 
Appendix A: Newcastle-Ottawa scale and quality data.

Supplemental Table 1: Newcastle-Ottawa Scale and quality data.

\begin{tabular}{|c|c|c|c|c|c|c|c|c|c|c|}
\hline \multirow{3}{*}{$\begin{array}{l}\text { Publica- } \\
\text { tion }\end{array}$} & \multirow[t]{3}{*}{ Year } & \multicolumn{9}{|c|}{ Newcastle-Ottawa Scale } \\
\hline & & \multicolumn{4}{|c|}{ Selection } & \multirow{2}{*}{$\begin{array}{l}\text { Compa- } \\
\text { rability }\end{array}$} & \multicolumn{3}{|c|}{ Outcome } & \multirow[t]{2}{*}{ Total } \\
\hline & & $\begin{array}{l}\text { Represen- } \\
\text { tativeness } \\
\text { of exposed } \\
\text { cohort }\end{array}$ & $\begin{array}{l}\text { Selection of } \\
\text { the non-ex- } \\
\text { posed co- } \\
\text { hort }\end{array}$ & $\begin{array}{l}\text { Ascer- } \\
\text { tainment } \\
\text { of expo- } \\
\text { sure }\end{array}$ & $\begin{array}{l}\text { Outcome } \\
\text { demon- } \\
\text { stration } \\
\text { at start }\end{array}$ & & $\begin{array}{l}\text { Assess- } \\
\text { ment of } \\
\text { outcome }\end{array}$ & $\begin{array}{l}\text { Follow-up } \\
\text { long enough } \\
\text { for outcome } \\
\text { to occur }\end{array}$ & $\begin{array}{l}\text { Ade- } \\
\text { quacy } \\
\text { of fol- } \\
\text { low-up }\end{array}$ & \\
\hline El Azeem & 2013 & * & * & * & * & ** & * & * & * & 9 \\
\hline Hamaguchi & 2007 & * & * & * & * & $* *$ & * & * & * & 9 \\
\hline Haring & 2009 & * & * & * & * & $* *$ & * & * & * & 9 \\
\hline Hwang & 2008 & * & * & * & * & $* *$ & * & * & * & 9 \\
\hline Lee & 2006 & * & * & * & * & $* *$ & * & * & * & 9 \\
\hline Lu & 2009 & * & * & * & * & $* *$ & * & * & * & 9 \\
\hline Ruttmann & 2005 & * & * & * & * & $* *$ & * & * & * & 9 \\
\hline Stepanova & 2012 & * & * & * & * & $* *$ & * & * & * & 9 \\
\hline Yu & 2008 & * & * & * & * & $* *$ & * & * & * & 9 \\
\hline Zheng & 2018 & * & * & * & * & $* *$ & * & * & * & 9 \\
\hline
\end{tabular}

The Newcastle-Ottawa Scale (NOS) evaluates the included studies based on selection, comparability and outcome. The maximum score for each criterion is 4,2 and 3 , respectively, with the maximum total score equaling 9 .

Supplemental Table 2: Quality data for eligible data sets.

\begin{tabular}{|l|l|l|l|l|l|l|l|l|l|l|}
\hline $\begin{array}{l}\text { Publica- } \\
\text { tion }\end{array}$ & Year & $\begin{array}{l}\text { Objec- } \\
\text { tive de- } \\
\text { fined }\end{array}$ & $\begin{array}{l}\text { Out- } \\
\text { come } \\
\text { de- } \\
\text { scribed }\end{array}$ & $\begin{array}{l}\text { Character- } \\
\text { istics de- } \\
\text { scribed }\end{array}$ & $\begin{array}{l}\text { Confound- } \\
\text { ers de- } \\
\text { scribed }\end{array}$ & $\begin{array}{l}\text { Main } \\
\text { find- } \\
\text { ings } \\
\text { out- } \\
\text { lined }\end{array}$ & $\begin{array}{l}\text { Heterog- } \\
\text { enous } \\
\text { popula- } \\
\text { tion }\end{array}$ & $\begin{array}{l}\text { Individuals } \\
\text { generating } \\
\text { data blind- } \\
\text { ed to out- } \\
\text { comes }\end{array}$ & $\begin{array}{l}\text { Repro- } \\
\text { ducibility } \\
\text { assessed }\end{array}$ & $\begin{array}{l}\text { Recruiting } \\
\text { all sub- } \\
\text { jects over } \\
\text { same time } \\
\text { period }\end{array}$ \\
\hline El Azeem & 2013 & Yes & Yes & Yes & Yes & Yes & No & NS & Yes \\
\hline $\begin{array}{l}\text { Hamagu- } \\
\text { chi }\end{array}$ & 2007 & Yes & Yes & Yes & Yes & Yes & No & Yes & Yes \\
\hline Haring & 2009 & Yes & Yes & Yes & Yes & Yes & No & Yes & Yes \\
\hline Hwang & 2008 & Yes & Yes & Yes & Yes & Yes & No & Yes & Yes \\
\hline Lee & 2006 & Yes & Yes & Yes & Yes & Yes & No & Yes & Yes \\
\hline Lu & 2009 & Yes & Yes & Yes & Yes & Yes & No & Yes & Yes \\
\hline Ruttmann & 2005 & Yes & Yes & Yes & Yes & Yes & No & Yes & Yes \\
\hline $\begin{array}{l}\text { Stepano- } \\
\text { va }\end{array}$ & 2012 & Yes & Yes & Yes & Yes & Yes & No & Yes & Yes \\
\hline Yu & 2008 & Yes & Yes & Yes & Yes & Yes & No & Yes & Yes \\
\hline Zheng & 2018 & Yes & Yes & Yes & Yes & Yes & No & Yes & Yes \\
\hline
\end{tabular}

NS: Not specified 


\section{Main results for Model 1, Random effects (MM), Z-Distribution, Log risk ratio}

$\begin{array}{lrcrrrr}\text { Covariate } & \text { Coefficient } & \begin{array}{c}\text { Standard } \\ \text { Error }\end{array} & \begin{array}{c}\mathbf{9 5 \%} \\ \text { Lower }\end{array} & \begin{array}{c}\mathbf{9 5 \%} \\ \text { Upper }\end{array} & \text { Z-value } & \begin{array}{c}\text { 2-sided } \\ \text { P-value }\end{array} \\ \text { Intercept } & -3.1739 & 0.6451 & -4.4382 & -1.9096 & -4.92 & 0.0000 \\ \text { Age } & 0.0518 & 0.0127 & 0.0270 & 0.0766 & 4.10 & 0.0000\end{array}$

\section{Statistics for Model 1}

Test of the model: Simultaneous test that all coefficients (excluding intercept) are zero

$\mathrm{Q}=16.77, \mathrm{df}=1, \mathrm{p}=0.0000$

Goodness of fit: Test that unexplained variance is zero

$\operatorname{Tau}^{2}=0.0251$, Tau $=0.1583, \mathrm{I}^{2}=66.33 \%, \mathrm{Q}=23.76, \mathrm{df}=8, \mathrm{p}=0.0025$

Comparison of Model 1 with the null model

Total between-study variance (intercept only)

$\mathrm{Tau}^{2}=0.1956$, Tau $=0.4422, \mathrm{I}^{2}=96.82 \%, \mathrm{Q}=282.66, \mathrm{df}=9, \mathrm{p}=0.0000$

Proportion of total between-study variance explained by Model 1

$R^{2}$ analog $=0.87$

Number of studies in the analysis $\quad \mathbf{1 0}$

Appendix B: For meta regression.

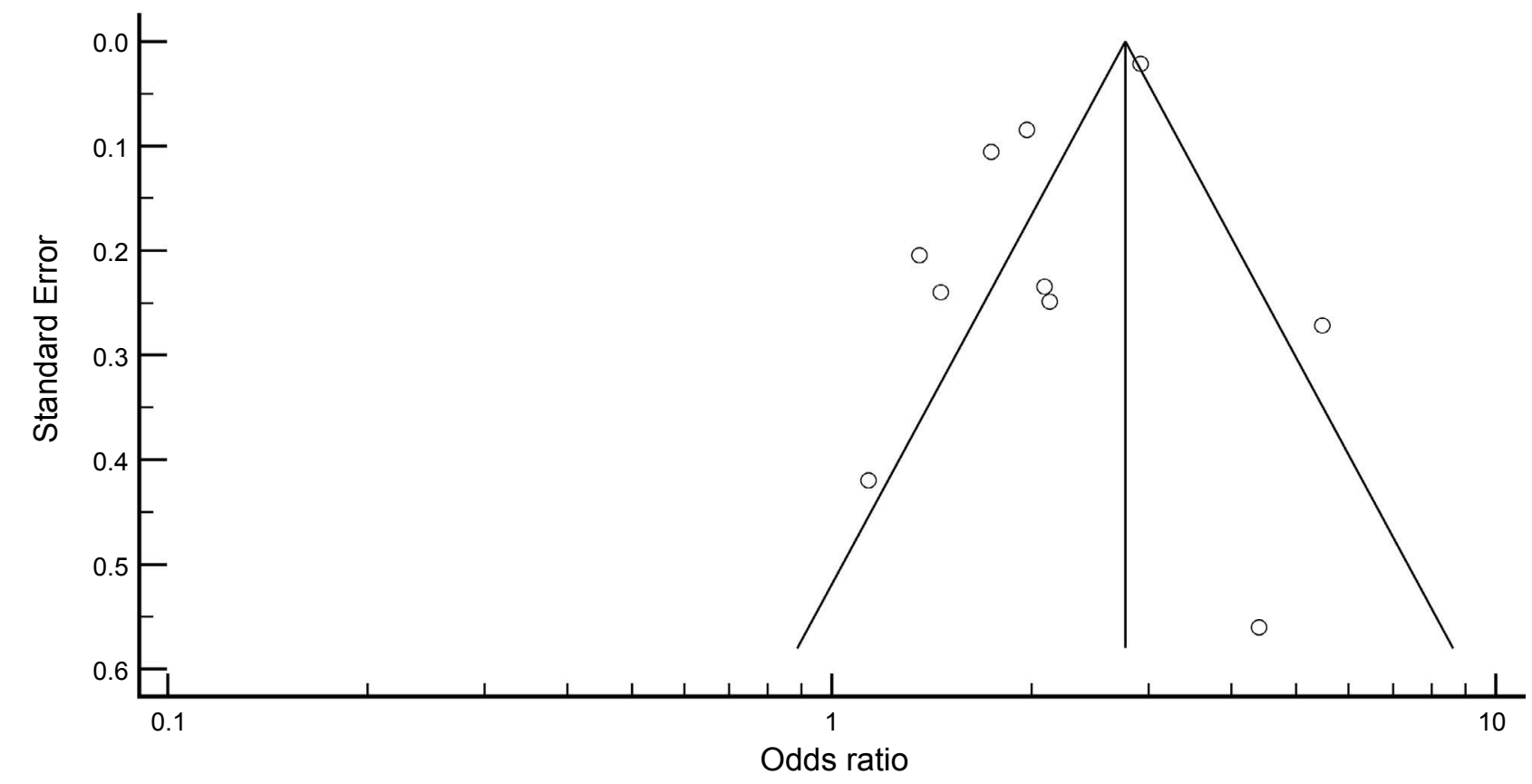

Appendix C: Funnel plot.

Funnel plot analysis to assess for potential publication bias and/or presence of heterogeneity for CV mortality and events. 


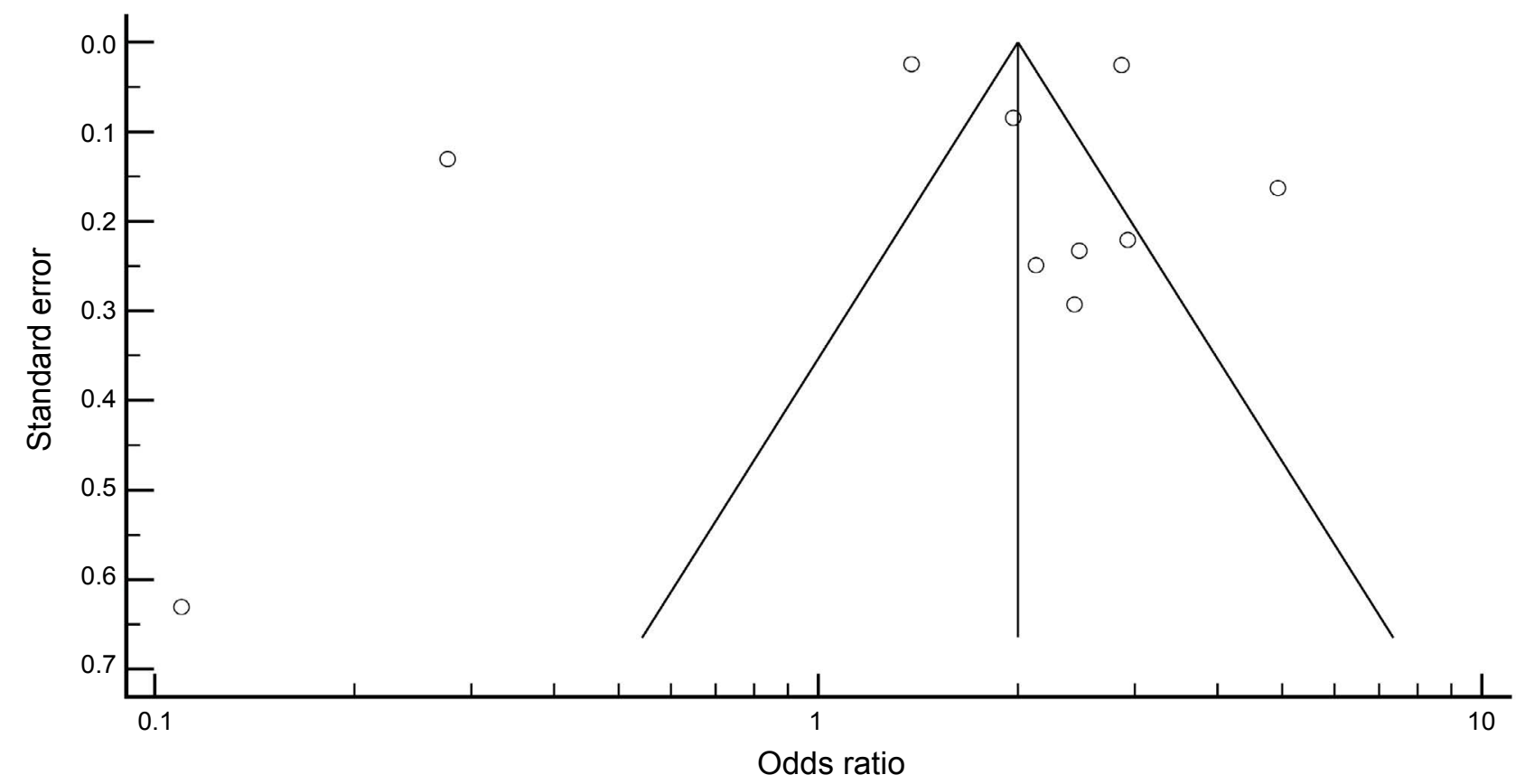

Appendix D: Funnel plot.

Funnel plot analysis to assess for potential publication bias and/or presence of heterogeneity for all-cause mortality.

\section{Funnel plot of standard error by Log risk ratio}

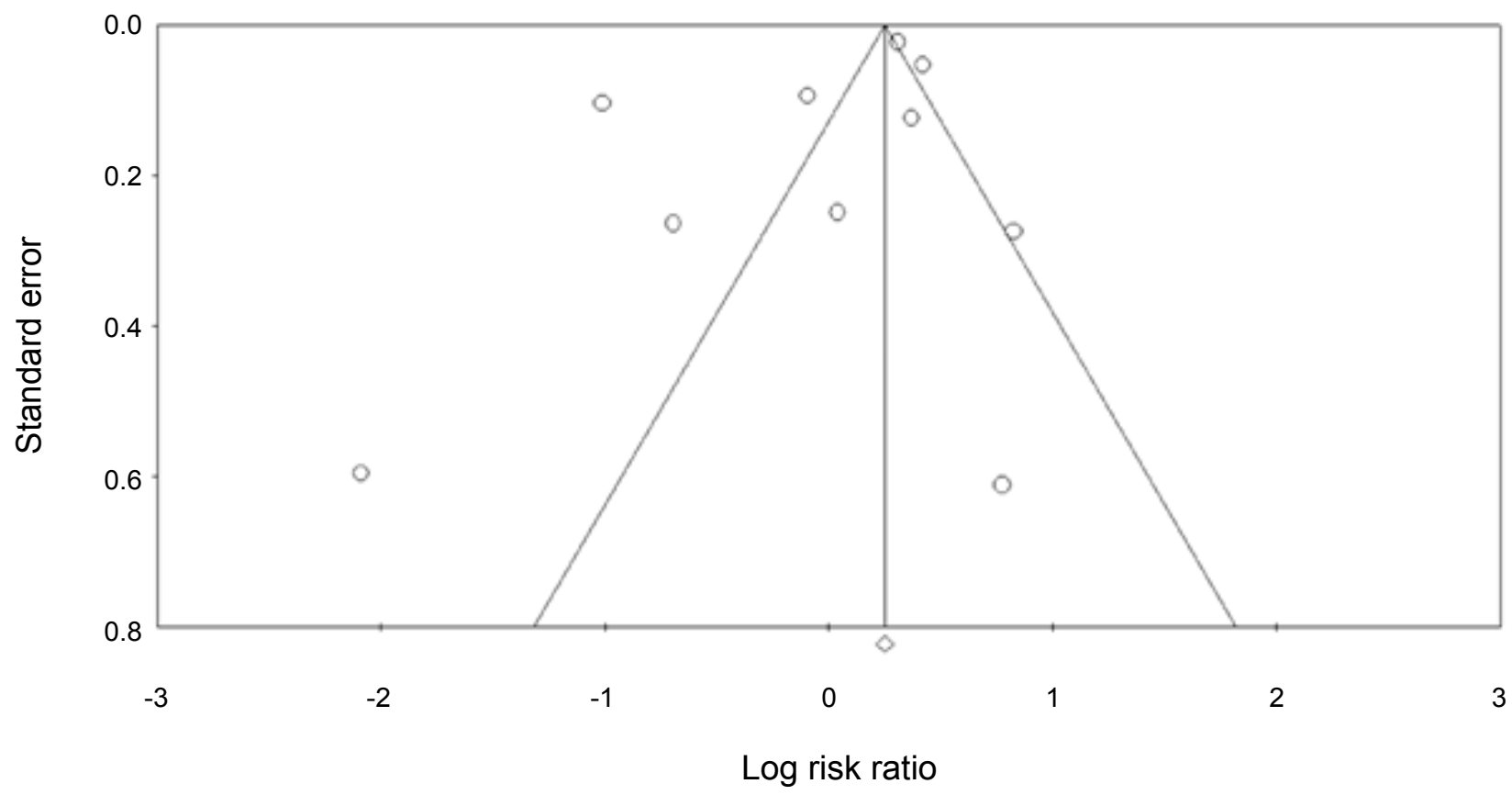

Appendix E: Funnel plot.

Funnel plot analysis to assess for potential publication bias and/or presence of heterogeneity for meta regression. 